\title{
Facile synthesis and antioxidant activity of lignin-related imidazo[1,2-a]pyridine derivatives
}

\author{
Xiaohui Yang, ${ }^{a, b}$ Qianqian Shang, ${ }^{* b}$ Caiying Bo, ${ }^{b}$ Lihong Hu, ${ }^{b}$ and Yonghong Zhou ${ }^{a, b}$ \\ ${ }^{a}$ Research Institute of Forestry New Technology, Chinese Academy of Forestry, Dongxiaofu-1 Xiangshan Road, \\ Beijing 100091, People's Republic of China \\ ${ }^{b}$ Key Lab. of Biomass Energy and Material, Institute of Chemical Industry of Forest Products, China Academy of \\ Forestry; Nanjing 210042, People's Republic of China \\ Email: shangqianqian@icifp.cn
}

Received 02-11-2018

Accepted 04-17-2018

Published on line $06-17-2018$

\section{Abstract}

A convenient protocol is described for the preparation of lignin related imidazo[1,2-a] pyridine derivatives via three-component reactions among 2-aminopyridines, isocyanides and aromatic aldehydes obtained from lignin with excellent yields. Their in vitro antioxidant effects were evaluated by scavenging effect on 2,2diphenyl-1-picrylhydrazyl (DPPH) radical. The structure activity relationship (SAR) studies presented that the introduction of electron-donating group in imidazo[1,2-a]pyridine could increase the radical scavenging activity; On the contrary, the presence of electron-withdrawing might decrease the radical scavenging activity. In addition, cyclopentyl and cyclohexyl groups almost had the same influence on the antioxidant activity. Furthermore, the methoxy moiety had a significant impact on antioxidant capability, and hence syringyl imidazo[1,2-a]pyridines $\mathbf{a}, \mathbf{d}, \mathbf{g}, \mathbf{j}$ and $\mathbf{m}$ exhibited excellent antioxidant properties

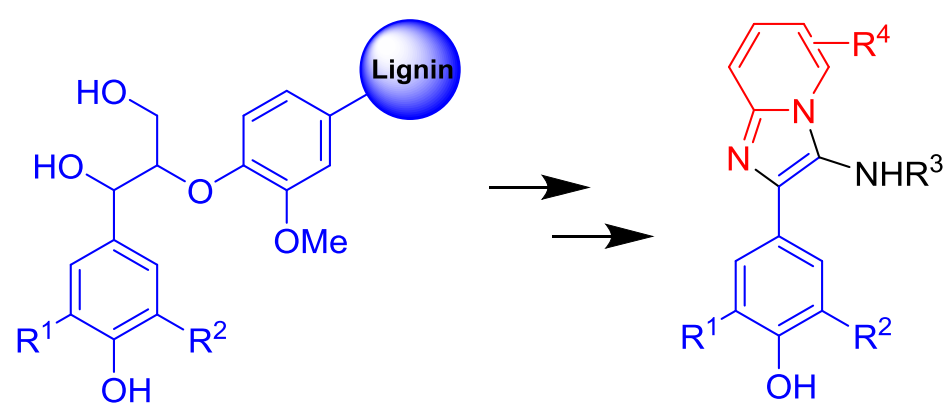

Keywords: Lignin; Imidazo[1,2-a]pyridine; Multi-component reactions; Antioxidant activity 


\section{Introduction}

Driven by the inevitable depletion of fossil fuels and the growing environmental problems, lignocellulosic biomass has been recognized as a potential feedstock for energy production and the synthesis of high-value chemicals. ${ }^{1-3}$ Lignin is, next to cellulose, the main component of lignocellulosic biomass and the only renewable resource which is rich in natural aromatic structure, so it is an ideal alternative material to prepare aromatic compounds. However, it retains the least utilized biomass constituent because of its complex and heterogeneous macromolecular structure. ${ }^{3,4}$ Most of lignin is burning for heat and power production, it is a low-value and an energy-inefficient application. ${ }^{5,6}$ In addition, a large volume of lignin is produced annually by the paper industry, and the volume is expected to surge with the introduction of future biorefineries. Hence, it is highly desirable to develop efficient procedures to increase application of lignin. ${ }^{7}$ Moreover, converting lignin into value-added products is also critical to increase revenue and establish economically feasible biorefining processes. $^{8}$

Recently, producing added-value aromatic monomers (typically vanillin and syringaldehyde) ${ }^{5}$ and their related pharmaceutical chemicals ${ }^{6,9,10}$ from lignin has been the topic of many publication. Those aromatic monomers degraded from lignin and their derivatives afforded good antioxidant ${ }^{6}$, anti-inflammatory, hepatoprotective9, antihypertensive, choleretic, antipsychotic, antispasmodic properties etc (Bjørsvik and Liguori ${ }^{10}$. Furthermore, lignin itself has been also proved to be antioxidant ${ }^{11}$, protein adsorption ${ }^{12}$, metal adsorption ${ }^{13}$, UV protection ${ }^{14}$ and antimicrobial properties ${ }^{15}$. Therefore, there is considerable interest in converting lignin to these high-value potential drugs while, at the same time, disposing of a high-volume, lowvalue waste.

Imidazopyridine, one of the important fused bicyclic 5-6 heterocycles, exhibits a broad spectrum of biological activities such as antitumor, anti-apoptotic, hypnoselective, antibacterial, antifungal, antiviral, antiprotozoal, anti-inflammatory ${ }^{16}$ and antioxidant ${ }^{17}$. Moreover, imidazo[1,2-a]pyridine is considered as a "drug prejudice" moiety, because there are numerous clinical drugs that includes zolpidem, saripidem, olprinone, zolimidine, rifaximin and drug candidates ${ }^{18,19}$. Recently, 2-phenyl-imidazo[1,2-a]pyridine analogues (tubulin polymerization inhibitors) ${ }^{20}$, sulfonylhydrazone-substituted imidazo[1,2-a]pyridines (PI3 kinase p110 $\alpha$ inhibitors $)^{21}$ and the other imidazo-[1,2-a]pyridine derivatives acting on Nek2 ${ }^{22}$ and c-Met kinases ${ }^{23}$ have been extensively studied in the literature for their excellent bioactivity.

Reactive oxygen species (ROS) contributes to DNA damage which is regarded to be the reason for disturbing healthy status and arising diseases ${ }^{24}$. The regulation of the in vivo oxidative stress might be effective therapeutic strategy for cancer and other diseases. Thus, discovering antioxidants from synthetic and natural compounds facilitated the treatment for ROS caused diseases, such as Alzheimer, pulmonary fibrosis and cancer etc. ${ }^{17}$ Inspired by the excellent antioxidant activity of lignin and imidazo[1,2-a]pyridine derivatives, it would thus be considering beneficial to synthesize lignin inspired imidazo[1,2-a]pyridine derivatives (Scheme 1) and evaluate their antioxidant activity. 


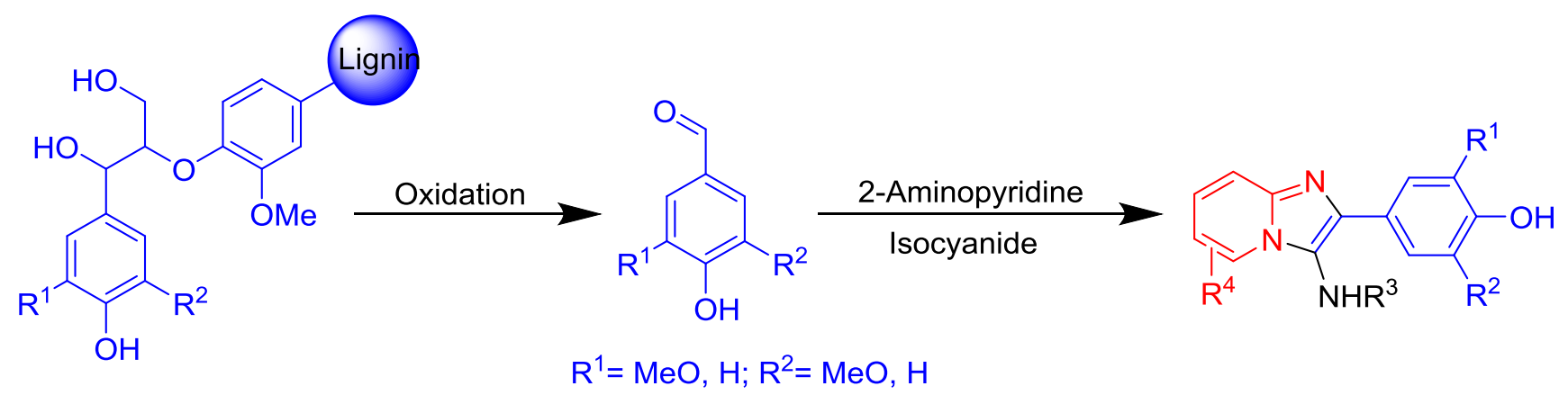

Scheme 1. Synthesis of lignin inspired imidazo[1,2-a]pyridine derivatives

\section{Result and Discussion}

\section{Chemistry}

As shown in Scheme 1, the aromatic aldehydes from lignin were prepared by nitrobenzene oxidation according to the reported method to provide vanillin, syringaldehyde and $p$-hydroxybenzaldehyde as the major compounds with total yield $10-17 \% .^{6}$ In addition, a highly efficient synthesis protocol of imidazo[1,2a]pyridines has been reported using Groebke three-component-reaction (3CR) among 2-aminopyridine, aldehyde and cyclohexyl isocyanide with good yields. ${ }^{17,26}$ For fast construction of lignin related imidazo[1,2a]pyridines for drug screening, the three-component-reaction was selected synthesis method. However, hydroxy group might leaded to by-products, and hence, the reaction of 2-aminopyridine, cyclopentylisonitrile, and syringaldehyde was selected as a test reaction to optimize the reaction conditions (catalyst, solvent and reaction time) (Table 1 ).

Table 1. Variation of reaction conditions for synthesis of 4-(3-(cyclopentylamino)imidazo[1,2-a]pyridin-2-yl)2,6-dimethoxyphenol

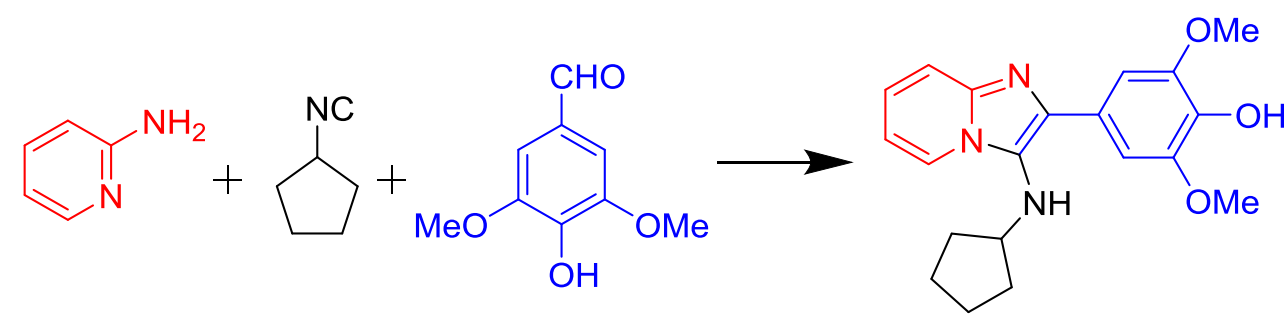

\begin{tabular}{ccccc}
\hline Entry & Catalyst & Solvent & Time $(\mathrm{h})$ & Yield (\%)a \\
\hline 1 & - & Water & 1 & 64 \\
2 & - & Water & 2 & 77 \\
3 & - & Water & 5 & 79 \\
4 & - & Water & overnight & 78 \\
5 & $\mathrm{NH}_{4} \mathrm{Cl}$ & Water & 2 & 88 \\
6 & $\mathrm{NH}_{4} \mathrm{Cl}$ & Toluene & 2 & 81 \\
7 & $\mathrm{NH}_{4} \mathrm{Cl}$ & Methanol & 2 & 88 \\
8 & $\mathrm{NH}_{4} \mathrm{Cl}$ & 1,4-Dioxane & 2 & 82 \\
\hline
\end{tabular}

${ }^{\mathrm{a}}$ Isolated yield 
We tried to synthesize the imidazo[1,2-a]pyridines in water using stainless steel autoclave with a Tefloncoated at $75^{\circ} \mathrm{C}$ in different time. We found that the yield was improved as the increase of reaction time, but increased a little after $2 \mathrm{~h}$; there was better yield and needed less time than that of reflux or room temperature in this approach. In order to further optimization of the reaction conditions, $\mathrm{NH}_{4} \mathrm{Cl}$ was selected as a catalyst because it is inexpensive and easy to be removed, and various solvents were also changed in presence of $\mathrm{NH}_{4} \mathrm{Cl}$. It was found that the yield increased by $11 \%$ in water in presence of $\mathrm{NH}_{4} \mathrm{Cl}$ within $2 \mathrm{~h}$, and the reactions in water or methanol were better than that in toluene and 1,4-dioxane. So the reaction was conducted in water or methanol with $\mathrm{NH}_{4} \mathrm{Cl}$ as a catalyst within $2 \mathrm{~h}$.

As indicated in Table 2, the three-component reaction was performed under water or methanol with $\mathrm{NH}_{4} \mathrm{Cl}$ as a catalyst at $75^{\circ} \mathrm{C}$ in $2 \mathrm{~h}$ using stainless steel autoclave with a Teflon-coated in high yields (85-94\%) in accordance with optimized reaction conditions. The structures of all synthesized products were identified by $\mathrm{IR},{ }^{1} \mathrm{H} N \mathrm{NMR},{ }^{13} \mathrm{C} N M R, \mathrm{MS}$ and $\mathrm{EA}$.

Table 2 Synthesis of imidazo[1,2-a]pyridine derivatives a-s

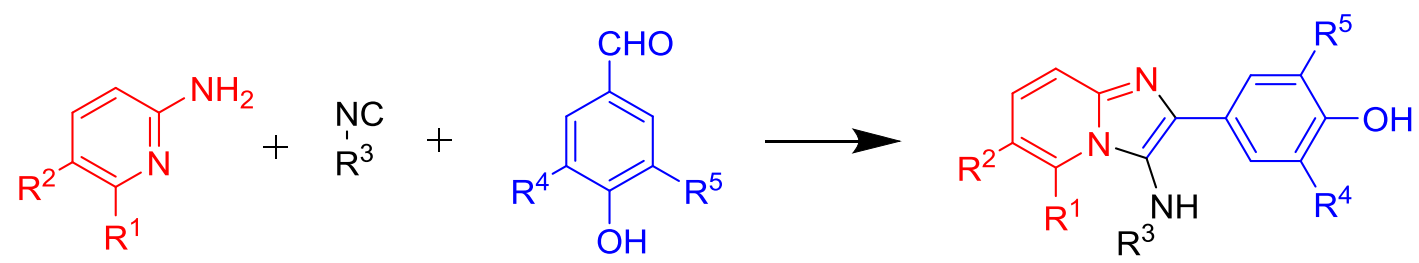

\begin{tabular}{cccccccc}
\hline Entry & Product & $\mathrm{R}^{1}$ & $\mathrm{R}^{2}$ & $\mathrm{R}^{3}$ & $\mathrm{R}^{4}$ & $\mathrm{R}^{5}$ & Yield (\%)* $^{*}$ \\
\hline 1 & a & $\mathrm{H}$ & $\mathrm{H}$ & Cyclopentyl & OMe & OMe & 88 \\
2 & $\mathbf{b}$ & $\mathrm{H}$ & $\mathrm{H}$ & Cyclopentyl & $\mathrm{H}$ & OMe & 84 \\
3 & $\mathbf{C}$ & $\mathrm{H}$ & $\mathrm{H}$ & Cyclopentyl & $\mathrm{H}$ & $\mathrm{H}$ & 89 \\
4 & $\mathbf{d}$ & $\mathrm{H}$ & $\mathrm{Me}$ & Cyclopentyl & OMe & OMe & 87 \\
5 & $\mathbf{e}$ & $\mathrm{H}$ & $\mathrm{Me}$ & Cyclopentyl & $\mathrm{H}$ & OMe & 86 \\
6 & $\mathbf{f}$ & $\mathrm{H}$ & $\mathrm{Me}$ & Cyclopentyl & $\mathrm{H}$ & $\mathrm{H}$ & 94 \\
7 & $\mathbf{g}$ & $\mathrm{H}$ & $\mathrm{H}$ & Cyclohexyl & OMe & OMe & 87 \\
8 & $\mathbf{H}$ & $\mathrm{H}$ & $\mathrm{H}$ & Cyclohexyl & $\mathrm{H}$ & OMe & 83 \\
9 & $\mathbf{i}$ & $\mathrm{H}$ & $\mathrm{H}$ & Cyclohexyl & $\mathrm{H}$ & $\mathrm{H}$ & 91 \\
10 & $\mathbf{j}$ & $\mathrm{H}$ & $\mathrm{Me}$ & Cyclohexyl & OMe & OMe & 87 \\
11 & $\mathbf{k}$ & $\mathrm{H}$ & $\mathrm{Me}$ & Cyclohexyl & $\mathrm{H}$ & $\mathrm{OMe}$ & 91 \\
12 & $\mathbf{I}$ & $\mathrm{H}$ & $\mathrm{Me}$ & Cyclohexyl & $\mathrm{H}$ & $\mathrm{H}$ & 84 \\
13 & $\mathbf{m}$ & $\mathrm{Br}$ & $\mathrm{Me}$ & Cyclopentyl & OMe & OMe & 88 \\
14 & $\mathbf{n}$ & $\mathrm{Br}$ & $\mathrm{Me}$ & Cyclohexyl & $\mathrm{H}$ & OMe & 86 \\
\hline
\end{tabular}

${ }^{\mathrm{a}}$ Isolated yield

\section{Antioxidant activity studies}

Scavenging of $N, N$-diphenyl- $N^{\prime}$-picrylhydrazyl (DPPH) free radical is the basis of a common antioxidant assay. So the antioxidant activity of the synthesized compounds was evaluated by DPPH assay. In our previous research, the synthesis and antioxidant activity of several lignin-related heterocyclic compounds (bis $(1 \mathrm{H}-$ pyrazole-5-ol)s, 1,4-dihydropyridines, polyhydroacridines, hexahydroquinolines and dihydro-pyrano[2,3c]pyrazoles) had been reported. ${ }^{6,27-29}$ The results showed that antioxidant activity of the compounds with syringyl (4-hydroxy-3,5-dimethoxyphenyl) moiety is greater than that of the compounds with guaiacyl (4- 
hydroxy-3-methoxyphenyl) moiety and much greater than that of the compounds with p-hydroxyphenyl moiety, i. e., the presence of methoxy group in aromatic heterocycles increase their antioxidant properties. The reason is that oxygen atom of ortho- $\mathrm{OCH}_{3}$ on hydroxyphenyl group have lone pair of electrons which can form big $p, \pi$-conjugation system with benzene ring and phenol hydroxyl radical, and $p, \pi$-conjugation can stabilize the phenol hydroxyl radical to promote free radical reaction. So the hydroxyphenyl moiety of aromatic heterocycles contains more ortho- $\mathrm{OCH}_{3}$, their antioxidant activity will be much better. Based on above rule, syringyl imidazo[1,2-a] pyridine a, guaiacyl imidazo[1,2-a] pyridine $\mathbf{b}$ and $p$-hydroxyphenyl imidazo[1,2-a] pyridine $c$ were hence chosen to be first evaluated.

As shown in Figure 1, the percentage inhibition of a was found to be best and that of $\mathbf{b}$ was found be good to moderate, while that of $\mathbf{c}$ was found to be relatively poor as compared to the standard Trolox. Especially, antioxidant activity of compound c containing two methoxy groups was almost equal to that of the standard Trolox. Evidently, the antioxidant activity of these compounds also follows the rule: Syringyl imidazo[1,2-a] pyridine > guaiacyl imidazo[1,2-a] pyridine > $p$-hydroxyphenyl imidazo[1,2-a] pyridine. In term of the result, the compounds $\mathbf{d}, \mathbf{g}, \mathbf{j}$ and $\mathbf{m}$ was next screened for their antioxidant activity. Comparing compounds $\mathbf{a}, \mathbf{g}$ with $\mathbf{d}$, j, introduction of methyl moiety increased their antioxidant property lightly. Meanwhile, the influence of cyclopentyl and cyclohexyl group almost made no difference on their antioxidant activity, for example, a and $\mathbf{g}$ had almost the same antioxidant activity, so $\mathbf{d}$ and $\mathbf{j}$ did. In addition, the presence of bromo group in aromatic heterocycles decreased the antioxidant performance because of electron-withdrawing effect. Above all, syringyl imidazo[1,2-a] pyridines $\mathbf{a}, \mathbf{d}, \mathbf{g}$, $\mathbf{j}$ and $\mathbf{m}$ exhibited excellent antioxidant activity. The strong antioxidant abilities of these compounds indicated their potential usefulness in the drug development, thus providing an effective way for value-added application of lignin.

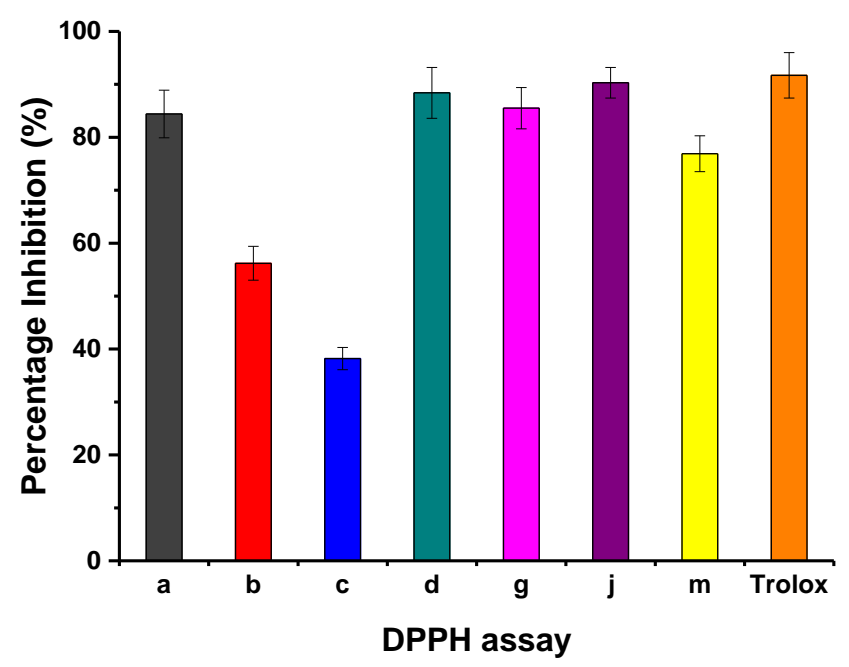

Figure 1. Radical scavenging activity in DPPH assay. Results were expressed as Percentage Inhibition

\section{Conclusions}

A convenient and efficient protocol was designed for synthesis of lignin related imidazo[1,2-a]pyridine derivatives under water or methanol with $\mathrm{NH}_{4} \mathrm{Cl}$ as a catalyst at $75^{\circ} \mathrm{C}$ in $2 \mathrm{~h}$ using stainless steel autoclave with 
a Teflon-coated. All compounds had good yields ( $\geq 83 \%)$. The antioxidant activity was evaluated by DPPH radical scavenging capability. The results indicated that antioxidant property of syringyl imidazo[1,2-a]pyridine was the best; that of guaiacyl imidazo[1,2-a]pyridines was good to moderate; and that of $p$-hydroxyphenyl imidazo[1,2-a]pyridine was relatively poor as compared to the standard Trolox. In addition, SAR studies showed that the introduction of electron-donating group in imidazo[1,2-a]pyridine could increase the radical scavenging activity. On the contrary, the presence of electron-withdrawing might decrease the radical scavenging activity. In addition, the effect of cyclopentyl and cyclohexyl moieties almost made no difference on the antioxidant activity.

\section{Experimental Section}

\section{Materials and methods}

Cyclohexylisonitrile, cyclopentylisonitrile, ${ }^{25}$ syringaldehyde, vanillin and $p$-Hydroxybenzaldehyde were synthesized according to the reported method. ${ }^{6}$ 2,2-Diphenyl-1-picrylhydrazyl (DPPH) was bought from Alfa Aesar. 2-Aminopyridine, methanol, ammonium chloride and the other reagents were obtained from Sinopharm Chemical Reagent Co, Ltd. All synthesized compounds were characterized by IR, ${ }^{1} \mathrm{H} N M R,{ }^{13} \mathrm{C} N M R$, MS and EA. IR spectra were recorded with a Nicolet Magna-IR 550 spectrometer. Mass spectra were recorded on an Agilent $1100 \mathrm{HPLC} / \mathrm{MS}$ coupled with a TSQ Quantum Ultra AM mass spectrometer using electrospray ionization (ESI). ${ }^{1} \mathrm{H}$ and ${ }^{13} \mathrm{C}$ NMR spectra were recorded with a BRUKER DRX-300 AVANCE spectrometer at 300 $\mathrm{MHz}$ and $75 \mathrm{MHz}$, respectively. Elemental analyses (C, H, N, S) were conducted using PE-2400(II) Elemental Analyser, their results were found to be in good agreement $( \pm 0.2 \%)$ with the calculated values. The UV absorbance was measured by Perkin Elmer Lambda 2 Spectrophotometer.

\section{Typical procedure for synthesis of lignin related imidazo[1,2-a]pyridines}

To a solution of $0.052 \mathrm{~g}$ ammonium chloride $(1 \mathrm{mmol})$ in $4 \mathrm{~mL}$ water or methanol was added isocyanide (1.2 $\mathrm{mmol})$, aromatic aldehyde $(1.1 \mathrm{mmol})$, and 2-aminopyridine $(1 \mathrm{mmol})$ and sealed in stainless steel autoclave with a Teflon-coated, and then the autoclave was put in the oven at $75^{\circ} \mathrm{C}$ in $2 \mathrm{~h}$. The autoclave was taken out and cooled to room temperature. The reaction mixture was put into $30 \mathrm{~mL}$ cooled water to afford the product as a precipitate. The solid residue was filtered and crystallized from ethyl acetate to give products.

4-(3-(cyclopentylamino)imidazo[1,2-a]pyridin-2-yl)-2,6-dimethoxyphenol. (a, $\left.\mathrm{C}_{20} \mathrm{H}_{24} \mathrm{~N}_{3} \mathrm{O}_{3}\right)(0.30 \mathrm{~g}$, 88\%). IR $\left.(\mathrm{KBr}) \mathrm{v} / \mathrm{cm}^{-1}: 3275,3092,2948,2927,2866,2828,1584,1502,1116,740 ;{ }^{1} \mathrm{H} \mathrm{NMR}(300 \mathrm{MHz}, \mathrm{CDCl})_{3}\right) \delta / \mathrm{ppm}$ : $8.10(\mathrm{~d}, 1 \mathrm{H}, \mathrm{J}=6.6 \mathrm{~Hz}), 7.54(\mathrm{~d}, 1 \mathrm{H}, \mathrm{J}=9.0 \mathrm{~Hz}), 7.38(\mathrm{~s}, 2 \mathrm{H}), 7.15(\mathrm{t}, 1 \mathrm{H}, \mathrm{J}=7.8 \mathrm{~Hz}), 6.80(\mathrm{t}, 1 \mathrm{H}, \mathrm{J}=6.6 \mathrm{~Hz}), 3.96(\mathrm{~s}$, $6 \mathrm{H}), 3.65-3.72(\mathrm{~m}, 1 \mathrm{H}), 1.40-1.87(\mathrm{~m}, 8 \mathrm{H}) ;{ }^{13} \mathrm{CNMR}\left(75.5 \mathrm{MHz}, \mathrm{CDCl}_{3}\right) \delta / \mathrm{ppm}: 147.1,141.3,137.2,134.5,124.7$, 124.2, 122.4, 117.1, 111.8, 104.3, 59.1, 56.4, 33.6, 23.7; LC-ESI-MS m/z: $354.2[\mathrm{M}+\mathrm{H}]^{+}$; Anal. Calcd for $\mathrm{C}_{20} \mathrm{H}_{23} \mathrm{~N}_{3} \mathrm{O}_{3}$ : C, 67.97; $\mathrm{H}, 6.56 ; \mathrm{N}, 11.89$; found: $\mathrm{C}, 67.90 ; \mathrm{H}, 6.65 ; \mathrm{N}, 11.94$.

4-(3-(cyclopentylamino)imidazo[1,2-a]pyridin-2-yl)-2-methoxyphenol. (b, $\left.\mathrm{C}_{19} \mathrm{H}_{21} \mathrm{~N}_{3} \mathrm{O}_{2}\right) 0.27 \mathrm{~g}(84 \%)$. IR (KBr) $v / \mathrm{cm}^{-1}: 3237,2948,2868,1610,1504,1244,1168,1030,830,753 ;{ }^{1} \mathrm{H} \mathrm{NMR}\left(300 \mathrm{MHz}, \mathrm{CDCl}_{3}\right) \delta / \mathrm{ppm}: 8.13(\mathrm{~d}$, $1 \mathrm{H}, J=6.9 \mathrm{~Hz}), 7.69(\mathrm{~s}, 1 \mathrm{H}), 7.50-7.59(\mathrm{~m}, 2 \mathrm{H}), 7.15(\mathrm{t}, 1 \mathrm{H}, J=8.7 \mathrm{~Hz}), 6.98(\mathrm{~d}, 1 \mathrm{H}, J=8.7 \mathrm{~Hz}), 6.81(\mathrm{t}, 1 \mathrm{H}, J=6.9 \mathrm{~Hz})$, $3.96(\mathrm{~s}, 3 \mathrm{H}), 3.62-3.71(\mathrm{~m}, 1 \mathrm{H}), 1.40-1.85(\mathrm{~m}, 8 \mathrm{H}) ;{ }^{13} \mathrm{C} \mathrm{NMR}\left(75.5 \mathrm{MHz}, \mathrm{CDCl}_{3}\right) \delta / \mathrm{ppm}: 146.9,145.4,141.3$, 138.1, 124.8, 122.5, 120.1, 117.0, 114.3, 111.8, 110.3, 59.2, 56.2, 33.5, 23.7; LC-ESI-MS m/z: $324.2[\mathrm{M}+\mathrm{H}]^{+}$; Anal. Calcd for $\mathrm{C}_{19} \mathrm{H}_{21} \mathrm{~N}_{3} \mathrm{O}_{2}$ : C, 70.57; $\mathrm{H}, 6.55 ; \mathrm{N}, 12.99$; found: $\mathrm{C}, 70.50 ; \mathrm{H}, 6.65 ; \mathrm{N}, 12.92$.

4-(3-(cyclopentylamino)imidazo[1,2-a]pyridin-2-yl)phenol. (c, $\left.\mathrm{C}_{18} \mathrm{H}_{19} \mathrm{~N}_{3} \mathrm{O}\right) 0.26 \mathrm{~g}(89 \%) . \mathrm{IR}(\mathrm{KBr}) \mathrm{v} / \mathrm{cm}^{-1}: 3325$, 2956, 2920, 2845, 1610, 1505, 1271, 1230, 836, 755; ${ }^{1} \mathrm{H} \mathrm{NMR}\left(300 \mathrm{MHz}, \mathrm{CDCl}_{3}\right) \delta / \mathrm{ppm}: 8.15$ (d, $\left.1 \mathrm{H}, \mathrm{J}=6.9 \mathrm{~Hz}\right)$, 
$7.75(\mathrm{~d}, 2 \mathrm{H}, J=8.7 \mathrm{~Hz}), 7.60(\mathrm{~d}, 1 \mathrm{H}, \mathrm{J}=9.0 \mathrm{~Hz}), 7.17(\mathrm{t}, 1 \mathrm{H}, \mathrm{J}=9.0 \mathrm{~Hz}), 6.80-6.90(\mathrm{~m}, 3 \mathrm{H}), 3.58-3.66(\mathrm{~m}, 1 \mathrm{H}), 1.35-$ $1.80(\mathrm{~m}, 8 \mathrm{H}) ;{ }^{13} \mathrm{C} \mathrm{NMR}\left(75.5 \mathrm{MHz}, \mathrm{CDCl}_{3}\right) \delta / \mathrm{ppm}: 157.4,144.7,140.7,139.2,135.9,128.8,124.9,122.7,116.0$, 113.3, 110.0, 59.1, 33.4, 23.6; LC-ESI-MS m/z: $294.1[\mathrm{M}+\mathrm{H}]^{+}$; Anal. Calcd for $\mathrm{C}_{18} \mathrm{H}_{19} \mathrm{~N}_{3} \mathrm{O}: \mathrm{C}, 73.69 ; \mathrm{H}, 6.53 ; \mathrm{N}$, 14.32; found: $\mathrm{C}, 73.61 ; \mathrm{H}, 6.58 ; \mathrm{N}, 14.34$.

4-(3-(cyclopentylamino)-5-methylimidazo[1,2-a]pyridin-2-yl)-2,6-dimethoxyphenol. (d, $\left.\mathrm{C}_{21} \mathrm{H}_{25} \mathrm{~N}_{3} \mathrm{O}_{3}\right) 0.32 \mathrm{~g}$ (87\%). IR (KBr) v/cm ${ }^{-1}$ : 3303, 2954, 2920, 2856, 1591, 1508, 1113, 855, 775; ${ }^{1} \mathrm{H} \mathrm{NMR}\left(300 \mathrm{MHz}, \mathrm{CDCl}_{3}\right) \delta / \mathrm{ppm}$ $7.47(\mathrm{~d}, 1 \mathrm{H}, \mathrm{J}=9.3 \mathrm{~Hz}), 7.28(\mathrm{~s}, 2 \mathrm{H}), 7.06(\mathrm{t}, 1 \mathrm{H}, \mathrm{J}=8.1 \mathrm{~Hz}), 6.49(\mathrm{~d}, 1 \mathrm{H}, \mathrm{J}=6.6 \mathrm{~Hz}), 3.98(\mathrm{~s}, 6 \mathrm{H}), 3.40-3.58(\mathrm{~m}, 1 \mathrm{H})$, $2.96(\mathrm{~s}, 3 \mathrm{H}), 1.32-1.68(\mathrm{~m}, 8 \mathrm{H}) ;{ }^{13} \mathrm{C} N M R\left(75.5 \mathrm{MHz}, \mathrm{CDCl}_{3}\right) \delta / \mathrm{ppm}: 147.1,143.1,138.3,133.2,127.0,126.4$, 124.2, 115.7, 113.2, 105.6, 61.4, 56.5, 32.8, 23.8, 20.0; LC-ESI-MS m/z: $368.2[\mathrm{M}+\mathrm{H}]^{+}$; Anal. Calcd for $\mathrm{C}_{21} \mathrm{H}_{25} \mathrm{~N}_{3} \mathrm{O}_{3}$ : C, 68.64; $\mathrm{H}, 6.86 ; \mathrm{N}, 11.44$; found: $\mathrm{C}, 68.71 ; \mathrm{H}, 6.82 ; \mathrm{N}, 11.49$.

4-(3-(cyclopentylamino)-5-methylimidazo[1,2-a]pyridin-2-yl)-2-methoxyphenol. $\left(e, \mathrm{C}_{20} \mathrm{H}_{23} \mathrm{~N}_{3} \mathrm{O}_{2}\right) 0.29 \mathrm{~g}(86 \%)$. IR (KBr) v/cm ${ }^{-1}: 3237,2945,2923,2863,1615,1510,1032,826,764 ;{ }^{1} \mathrm{H} \mathrm{NMR}\left(300 \mathrm{MHz}, \mathrm{CDCl}_{3}\right) \delta / \mathrm{ppm}: 7.67$ (s, $1 \mathrm{H}), 7.53(\mathrm{~d}, 1 \mathrm{H}, J=8.4 \mathrm{~Hz}), 7.29$ (d, $1 \mathrm{H}, J=9.0 \mathrm{~Hz}), 7.03(\mathrm{t}, 1 \mathrm{H}, J=8.4 \mathrm{~Hz}), 6.82(\mathrm{~d}, 1 \mathrm{H}, J=8.4 \mathrm{~Hz}), 6.53$ (d, $1 \mathrm{H}, J=8.1$ $\mathrm{Hz}), 3.83(\mathrm{~s}, 3 \mathrm{H}), 3.38-3.49(\mathrm{~m}, 1 \mathrm{H}), 2.93(\mathrm{~s}, 3 \mathrm{H}), 1.30-1.58(\mathrm{~m}, 8 \mathrm{H}) ;{ }^{13} \mathrm{C} \mathrm{NMR}\left(75.5 \mathrm{MHz}, \mathrm{CDCl}_{3}\right) \delta / \mathrm{ppm}: 147.2$, 145.8, 141.9, 138.3, 136.3, 126.6, 126.3, 123.8, 120.3, 115.1, 114.8, 112.8, 111.5, 59.9, 55.6, 32.1, 23.5, 19.3; LC-ESI-MS m/z: 338.1 [M+H] ; Anal. Calcd for $\mathrm{C}_{20} \mathrm{H}_{23} \mathrm{~N}_{3} \mathrm{O}_{2}$ : C, 71.19; $\mathrm{H}, 6.87 ; \mathrm{N}, 12.45$; found: $\mathrm{C}, 71.11 ; \mathrm{H}, 6.92$; N, 12.40 .

4-(3-(cyclopentylamino)-5-methylimidazo[1,2-a]pyridin-2-yl)phenol. (f, $\left.\mathrm{C}_{19} \mathrm{H}_{21} \mathrm{~N}_{3} \mathrm{O}\right) 0.29 \mathrm{~g}(94 \%)$ IR (KBr) $v / \mathrm{cm}^{-1}: 3219,2951,2904,2862,1613,1504,1260,1227,1177,841,775 ;{ }^{1} \mathrm{H} \mathrm{NMR}\left(300 \mathrm{MHz}, \mathrm{CDCl}_{3}\right) \delta / \mathrm{ppm}:$ $7.60(\mathrm{~d}, 2 \mathrm{H}, J=8.4 \mathrm{~Hz}), 7.48(\mathrm{~d}, 1 \mathrm{H}, \mathrm{J}=8.7 \mathrm{~Hz}), 7.05(\mathrm{dd}, 1 \mathrm{H}, J=6.9,8.7 \mathrm{~Hz}), 6.73(\mathrm{~d}, 2 \mathrm{H}, J=8.4 \mathrm{~Hz}), 6.48(\mathrm{~d}, 1 \mathrm{H}$, $J=6.9 \mathrm{~Hz}), 3.22-3.62(\mathrm{~m}, 1 \mathrm{H}), 2.96(\mathrm{~s}, 3 \mathrm{H}), 1.25-1.57(\mathrm{~m}, 8 \mathrm{H}) ;{ }^{13} \mathrm{C} \mathrm{NMR}\left(75.5 \mathrm{MHz}, \mathrm{CDCl}_{3}\right) \delta / \mathrm{ppm}: 156.9,142.6$, 138.3, 136.6, 134.2, 129.4, 127.3, 124.8, 115.9, 114.9, 113.9, 61.4, 32.7, 23.7, 20.1; LC-ESI-MS m/z: 308.2 $[\mathrm{M}+\mathrm{H}]^{+}$; Anal. Calcd for $\mathrm{C}_{19} \mathrm{H}_{21} \mathrm{~N}_{3} \mathrm{O}: \mathrm{C}, 74.24 ; \mathrm{H}, 6.89 ; \mathrm{N}, 13.67$; found: $\mathrm{C}, 74.31 ; \mathrm{H}, 6.82 ; \mathrm{N}, 13.74$.

4-(3-(cyclohexylamino)imidazo[1,2-a]pyridin-2-yl)-2,6-dimethoxyphenol. (g, $\left.\mathrm{C}_{21} \mathrm{H}_{25} \mathrm{~N}_{3} \mathrm{O}_{3}\right) \quad 0.32 \mathrm{~g}$ (87\%). IR $(\mathrm{KBr}) \mathrm{v} / \mathrm{cm}^{-1}: 3325,2925,2848,1608,1520,1217,1109,760 ;{ }^{1} \mathrm{H}$ NMR $\left(300 \mathrm{MHz}, \mathrm{CDCl}_{3}\right) \delta / \mathrm{ppm}: 8.29$ (d, $1 \mathrm{H}$, $J=6.6 \mathrm{~Hz}$ ), $8.08(\mathrm{~d}, 1 \mathrm{H}, \mathrm{J}=6.6 \mathrm{~Hz}), 7.23(\mathrm{t}, 1 \mathrm{H}, \mathrm{J}=6.6 \mathrm{~Hz}), 6.96(\mathrm{~d}, 1 \mathrm{H}, \mathrm{J}=6.6 \mathrm{~Hz}), 6.64(\mathrm{~s}, 2 \mathrm{H}), 5.47(\mathrm{~s}, 1 \mathrm{H}), 3.80(\mathrm{~s}$, $6 \mathrm{H}), 2.86-2.98(\mathrm{~m}, 1 \mathrm{H}), 1.15-1.42(\mathrm{~m}, 10 \mathrm{H}) ;{ }^{13} \mathrm{C} \mathrm{NMR}\left(75.5 \mathrm{MHz}, \mathrm{CDCl}_{3}\right) \delta / \mathrm{ppm}: 146.5,141.1,135.4,134.6$, 126.5, 125.4, 122.6, 121.6, 117.2, 113.6, 106.4, 56.8, 56.6, 34.1, 25.5, 24.6; LC-ESI-MS m/z: 368.3 [M+H] ${ }^{+}$; Anal. Calcd for $\mathrm{C}_{21} \mathrm{H}_{25} \mathrm{~N}_{3} \mathrm{O}_{3}: \mathrm{C}, 68.64 ; \mathrm{H}, 6.86 ; \mathrm{N}, 11.44$; found: $\mathrm{C}, 68.71 ; \mathrm{H}, 6.80 ; \mathrm{N}, 11.48$.

4-(3-(cyclohexylamino)imidazo[1,2-a]pyridin-2-yl)-2-methoxyphenol. (h, $\left.\mathrm{C}_{20} \mathrm{H}_{23} \mathrm{~N}_{3} \mathrm{O}_{2}\right) 0.28 \mathrm{~g}(83 \%)$. IR (KBr) $v / \mathrm{cm}^{-1}: 3358,2927,2850,1600,1501,1408,1279,1030,753,740 ;{ }^{1} \mathrm{H}$ NMR (300 MHz, MeOD) $\delta / p p m: 8.31$ (d, $1 \mathrm{H}, J=6.9 \mathrm{~Hz}), 7.71(\mathrm{~s}, 1 \mathrm{H}), 7.54(\mathrm{~d}, 1 \mathrm{H}, \mathrm{J}=6.9 \mathrm{~Hz}), 7.45(\mathrm{t}, 1 \mathrm{H}, \mathrm{J}=6.9 \mathrm{~Hz}), 7.25(\mathrm{t}, 1 \mathrm{H}, J=6.9 \mathrm{~Hz}), 6.87-6.94(\mathrm{~m}, 2 \mathrm{H})$, $3.80(\mathrm{~s}, 3 \mathrm{H}), 2.93-3.00(\mathrm{~m}, 1 \mathrm{H}), 1.10-1.50(\mathrm{~m}, 10 \mathrm{H}) ;{ }^{13} \mathrm{C} N M R(75.5 \mathrm{MHz}, \mathrm{MeOD}) \delta / \mathrm{ppm}: 149.0,147.4,142.6$, 137.4, 127.2, 126.1, 125.8, 124.3, 121.5, 116.8, 116.2, 113.0, 112.2, 57.7, 56.5, 35.1, 27.0, 25.9; LC-ESI-MS m/z: $338.2[\mathrm{M}+\mathrm{H}]^{+}$; Anal. Calcd for $\mathrm{C}_{20} \mathrm{H}_{23} \mathrm{~N}_{3} \mathrm{O}_{2}$ : C, 71.19; $\mathrm{H}, 6.87 ; \mathrm{N}, 12.45$; found: $\mathrm{C}, 71.10 ; \mathrm{H}, 6.81 ; \mathrm{N}, 12.53$.

4-(3-(cyclohexylamino)imidazo[1,2-a]pyridin-2-yl)phenol. (i, $\left.\mathrm{C}_{19} \mathrm{H}_{21} \mathrm{~N}_{3} \mathrm{O}\right) 0.28 \mathrm{~g}(91 \%)$. IR $(\mathrm{KBr}) \mathrm{v} / \mathrm{cm}^{-1}: 3295$,

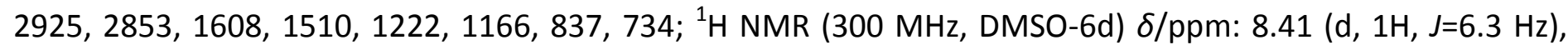
$7.74(\mathrm{t}, 1 \mathrm{H}, J=6.3 \mathrm{~Hz}), 7.52(\mathrm{t}, 2 \mathrm{H}, J=7.5 \mathrm{~Hz}), 7.01(\mathrm{t}, 1 \mathrm{H}, J=6.3 \mathrm{~Hz}), 6.90(\mathrm{~d}, 1 \mathrm{H}, J=6.3 \mathrm{~Hz}), 6.75(\mathrm{~d}, 2 \mathrm{H}, J=7.5 \mathrm{~Hz})$,

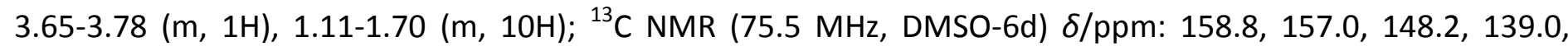
$131.8,131.5,128.7,116.6,110.8,56.7,33.3,26.5,26.1$; LC-ESI-MS m/z: 352.1 [M-H+2Na] ; Anal. Calcd for $\mathrm{C}_{19} \mathrm{H}_{21} \mathrm{~N}_{3} \mathrm{O}: \mathrm{C}, 74.24 ; \mathrm{H}, 6.89 ; \mathrm{N}, 13.67$; found: $\mathrm{C}, 74.31 ; \mathrm{H}, 6.82 ; \mathrm{N}, 13.61$.

4-(3-(cyclohexylamino)-5-methylimidazo[1,2-a]pyridin-2-yl)-2,6-dimethoxyphenol. (j, $\left.\mathrm{C}_{22} \mathrm{H}_{27} \mathrm{~N}_{3} \mathrm{O}_{3}\right) \quad 0.33$ g (87\%). IR (KBr) v/cm ${ }^{-1}: 3323,2950,2923,2851,1593,1513,1113,772 ;{ }^{1} \mathrm{H} N M R\left(300 \mathrm{MHz}, \mathrm{CDCl}_{3}\right) \delta / \mathrm{ppm}: 7.42$ (d, $1 \mathrm{H}, J=9.0 \mathrm{~Hz}), 7.31(\mathrm{~s}, 2 \mathrm{H}), 7.01(\mathrm{dd}, 1 \mathrm{H}, \mathrm{J}=6.9,9.0 \mathrm{~Hz}), 6.43(\mathrm{~d}, 1 \mathrm{H}, J=6.9 \mathrm{~Hz}), 3.98(\mathrm{~s}, 6 \mathrm{H}), 2.94(\mathrm{~s}, 3 \mathrm{H}), 2.75-$ 
$2.85(\mathrm{~m}, 1 \mathrm{H}), 1.10-1.75(\mathrm{~m}, 10 \mathrm{H}) ;{ }^{13} \mathrm{C} N M R\left(75.5 \mathrm{MHz}, \mathrm{CDCl}_{3}\right) \delta / \mathrm{ppm}: 146.0,141.3,139.2,138.7,133.5,132.1$, 126.6, 123.7, 114.5, 111.6, 103.9, 55.5, 55.2, 32.3, 24.4, 23.8, 19.2; LC-ESI-MS m/z: 382.2 [M+H] ${ }^{+}$; Anal. Calcd for $\mathrm{C}_{22} \mathrm{H}_{27} \mathrm{~N}_{3} \mathrm{O}_{3}$ : C, 69.27; $\mathrm{H}, 7.13 ; \mathrm{N}, 11.02$; found: $\mathrm{C}, 69.20 ; \mathrm{H}, 7.19 ; \mathrm{N}, 11.08$.

4-(3-(cyclohexylamino)-5-methylimidazo[1,2-a]pyridin-2-yl)-2-methoxyphenol. $\left(\mathbf{k}, \mathrm{C}_{21} \mathrm{H}_{25} \mathrm{~N}_{3} \mathrm{O}_{2}\right) 0.32 \mathrm{~g}(91 \%)$. IR (KBr) v/cm ${ }^{-1}: 3316,2930,2854,1560,1510,1415,1228,1034,821,779 ;{ }^{1} \mathrm{H} N M R(300 \mathrm{MHz}, \mathrm{CDCl} 3) \delta / \mathrm{ppm}:$ $7.62(\mathrm{~s}, 1 \mathrm{H}), 7.42(\mathrm{~d}, 1 \mathrm{H}, \mathrm{J}=6.3 \mathrm{~Hz}), 6.90-7.10(\mathrm{~m}, 3 \mathrm{H}), 6.47(\mathrm{~d}, 1 \mathrm{H}, \mathrm{J}=6.3 \mathrm{~Hz}), 3.95(\mathrm{~s}, 3 \mathrm{H}), 2.95(\mathrm{~s}, 3 \mathrm{H}), 2.70-2.83$ $(\mathrm{m}, 1 \mathrm{H}), 1.05-1.67(\mathrm{~m}, 10 \mathrm{H}) ;{ }^{13} \mathrm{C}$ NMR $\left(75.5 \mathrm{MHz}, \mathrm{CDCl}_{3}\right) \delta / \mathrm{ppm}: 146.7,145.2,139.1,136.3,129.6,125.9$, 124.2, 120.7, 115.5, 114.2, 113.5, 110.7, 58.6, 56.1, 33.3, 25.8, 24.9, 20.2; LC-ESI-MS m/z: 352.1 [M+H] ; Anal. Calcd for $\mathrm{C}_{21} \mathrm{H}_{25} \mathrm{~N}_{3} \mathrm{O}_{2}: \mathrm{C}, 71.77 ; \mathrm{H}, 7.17 ; \mathrm{N}, 11.96$; found: $\mathrm{C}, 71.85 ; \mathrm{H}, 7.11 ; \mathrm{N}, 11.92$.

4-(3-(cyclohexylamino)-5-methylimidazo[1,2-a]pyridin-2-yl)phenol. (I, $\left.\mathrm{C}_{20} \mathrm{H}_{23} \mathrm{~N}_{3} \mathrm{O}\right) 0.27 \mathrm{~g}(84 \%) . \mathrm{IR}(\mathrm{KBr}) \mathrm{v} / \mathrm{cm}$

${ }^{1}: 3270,2827,2854,1652,1596,1511,1452,1230,1165,841,778 ;{ }^{1} \mathrm{H} N M R\left(300 \mathrm{MHz}, \mathrm{CDCl}_{3}\right) \delta / \mathrm{ppm}: 7.60$ (d, $2 \mathrm{H}, J=8.7 \mathrm{~Hz}), 7.46(\mathrm{~d}, 1 \mathrm{H}, J=9.0 \mathrm{~Hz}), 7.03(\mathrm{dd}, 1 \mathrm{H}, J=6.9,9.0 \mathrm{~Hz}), 6.82(\mathrm{~d}, 1 \mathrm{H}, J=8.7 \mathrm{~Hz}), 6.46(\mathrm{~d}, 2 \mathrm{H}, J=6.9 \mathrm{~Hz})$, $2.95(\mathrm{~s}, 3 \mathrm{H}), 2.68-2.81(\mathrm{~m}, 1 \mathrm{H}), 1.12-1.52(\mathrm{~m}, 10 \mathrm{H}) ;{ }^{13} \mathrm{C} \mathrm{NMR}\left(75.5 \mathrm{MHz}, \mathrm{CDCl}_{3}\right) \delta / \mathrm{ppm}: 157.6,141.6,136.7$, 135.5, 132.4, 129.3, 128.7, 125.9, 116.2, 114.6, 111.4, 58.2, 32.9, 25.8, 24.9, 20.1; LC-ESI-MS m/z: 322.2 $[\mathrm{M}+\mathrm{H}]^{+}$; Anal. Calcd for $\mathrm{C}_{20} \mathrm{H}_{23} \mathrm{~N}_{3} \mathrm{O}: \mathrm{C}, 74.74 ; \mathrm{H}, 7.21 ; \mathrm{N}, 13.07$; found: $\mathrm{C}, 74.81 ; \mathrm{H}, 7.27 ; \mathrm{N}, 13.02$.

4-(6-bromo-3-(cyclopentylamino)-5-methylimidazo[1,2-a]pyridin-2-yl)-2,6-dimethoxyphenol. $\left(\mathrm{m}_{,} \mathrm{C}_{21} \mathrm{H}_{24} \mathrm{BrN}_{3} \mathrm{O}_{3}\right)$ $0.39 \mathrm{~g}(88 \%)$. IR (KBr) v/cm ${ }^{-1}: 3295,2951,2920,2851,1594,1510,1418,1224,1116,855,789 ;{ }^{1} \mathrm{H} N M R(300$ $\left.\mathrm{MHz}_{2} \mathrm{CDCl}_{3}\right) \delta / \mathrm{ppm}: 7.17-7.31(\mathrm{~m}, 4 \mathrm{H}), 3.95(\mathrm{~s}, 6 \mathrm{H}), 3.46-3.51(\mathrm{~m}, 1 \mathrm{H}), 2.96(\mathrm{~s}, 3 \mathrm{H}), 1.10-1.64(\mathrm{~m}, 8 \mathrm{H}) ;{ }^{13} \mathrm{C} \mathrm{NMR}$ $\left(75.5 \mathrm{MHz}, \mathrm{CDCl}_{3}\right) \delta / \mathrm{ppm}: 163.8,147.2,141.7,139.6,134.8,128.6,128.4,125.7,115.9,110.0,105.0,61.2$, 56.5, 32.7, 23.7, 18.2; LC-ESI-MS m/z: $446.1[\mathrm{M}+\mathrm{H}]^{+}$; Anal. Calcd for $\mathrm{C}_{21} \mathrm{H}_{24} \mathrm{BrN}_{3} \mathrm{O}_{3}$ : C, 56.51; $\mathrm{H}, 5.42 ; \mathrm{N}, 9.41$; found: C, 56.60; $\mathrm{H}, 5.34 ; \mathrm{N}, 9.45$.

4-(6-bromo-3-(cyclohexylamino)-5-methylimidazo[1,2-a]pyridin-2-yl)-2-methoxyphenol. (n, $\mathrm{C}_{21} \mathrm{H}_{24} \mathrm{BrN}_{3} \mathrm{O}_{2}$ ) $0.37 \mathrm{~g}$ (86\%). IR (KBr) v/cm ${ }^{-1}: 3293,2951,2920,2848,1593,1500,1402,1124,1032,816,785 ;{ }^{1} \mathrm{H}$ NMR (300 $\left.\mathrm{MHz}, \mathrm{CDCl}_{3}\right) \delta / p p m: 7.54(\mathrm{~s}, 1 \mathrm{H}), 7.32-7.36(\mathrm{~m}, 2 \mathrm{H}), 6.80-6.92(\mathrm{~m}, 2 \mathrm{H}), 3.98(\mathrm{~s}, 3 \mathrm{H}), 3.17-3.22(\mathrm{~m}, 1 \mathrm{H}), 3.16(\mathrm{~s}$, 3H), 1.00-1.70 (m, 10H); $\left.{ }^{13} \mathrm{C} \mathrm{NMR} \mathrm{(75.5} \mathrm{MHz,} \mathrm{CDCl}_{3}\right) \delta / \mathrm{ppm:} \mathrm{162.2,} \mathrm{147.6,} \mathrm{145.8,} \mathrm{142.3,} \mathrm{140.9,} \mathrm{138.4,} \mathrm{130.1,}$ 126.0, 126.2, 116.4, 114.5, 111.5, 110.1, 58.4, 56.2, 35.5, 25.0, 24.8, 18.6; LC-ESI-MS m/z: 430.2 [M+H] ${ }^{+}$; Anal. Calcd for $\mathrm{C}_{21} \mathrm{H}_{24} \mathrm{BrN}_{3} \mathrm{O}_{2}$ : C, 58.61; $\mathrm{H}, 5.62 ; \mathrm{N}, 9.76$; found: $\mathrm{C}, 58.70 ; \mathrm{H}, 5.68 ; \mathrm{N}, 9.71$.

\section{Antioxidant evaluation}

The free radical scavenging activity were evaluated by the 2,2'-diphenyl-1-picrylhydrazyl (DPPH) radical scavenging method, which is based on the measurement of the scavenging ability towards the stable 2,2diphenyl-1-picrylhydrazyl (DPPH) radical. The assay was conducted according to the reported method. ${ }^{6}$ Briefly, an ethanol solution $(200 \mu \mathrm{L})$ of $100 \mu \mathrm{M}$ diphenyl-picryl hydrazide was incubated at $30{ }^{\circ} \mathrm{C}$ with $1 \mu \mathrm{L}$ of compound solution in deionized $\mathrm{H}_{2} \mathrm{O}$. The final concentration of compounds was $100 \mu \mathrm{M}$. A decrease in absorbance was measured at $517 \mathrm{~nm}$. The absorbance was measured at $t=0 \mathrm{~min}\left(\mathrm{~A}_{0}\right)$ and after $10 \mathrm{~min}$ incubation at room temperature $\left(\mathrm{A}_{10}\right)$. The radical scavenging activity was expressed as Percentage Inhibition of DPPH free radical (I\%) and calculated according to the Eq. (1). All experiments were carried out in triplicate. The 6-hydroxyl-2,5,7,8-tetramethylchroman-2-carboxylic acid (Trolox) was used as antioxidant reference.

$$
\mathrm{I} \%=100 \times\left(\mathrm{A}_{0}-\mathrm{A}_{10}\right) / \mathrm{A}_{0}
$$




\section{Acknowledgements}

The authors are grateful for Fundamental Research Funds of Research Institute of Forestry New Technology, Grant. No. CAFYBB2016SY027 and Fundamental Research Funds from Jiangsu Province Biomass and Materials Laboratory, Grant. No. JSBEM-S-201803.

\section{References}

1. Rouches, E.; Herpoël-Gimbert, I.; Steyer, J. P.; Carrere, H. Renew. Sust. Energ. Rev. 2016, 59, 179-198. https://doi.org/10.1016/i.rser.2015.12.317

2. Shao, Y.; Xia, Q.; Dong, L.; Liu, X.; Han, X.; Parker, S. F.; Cheng, Y.; Daemen, L. L.; Ramirez-Cuesta, A. J.; Yang, S.; Wang Y. Nature Commun. 2017, 8, 1-9. 10.1038/ncomms16104

3. Yang, X.; Li, N.; Lin, X.; Pan, X.; Zhou, Y. J. Agric. Food Chem. 2016, 44, 8379-8387. 10.1021/acs.jafc.6b03807

4. Ma, X.; Ma, R.; Hao, W.; Chen, M.; Yan, F.; Cui, K.; Tian, Y.; Li, Y. ACS Catal. 2015, 5, 4803-4813. 10.1021/acscatal.5b01159

5. Rodrigues Pinto, P. C.; Borges da Silva, E. A.; Rodrigues, A. E. Ind. Eng. Chem. Res. 2011, 50, 741-748. 10.1021/ie102132a

6. Yang, X.; Zhang, P.; Wang, Z.; Jing, F.; Zhou, Y.; Hu, L. Ind. Crop. Prod. 2014, 52, 413-419. https://doi.org/10.1016/i.indcrop.2013.11.017

7. Deuss, P. J.; Barta, K. Coord. Chem. Rev. 2016, 306, 510-532. https://doi.org/10.1016/i.ccr.2015.02.004

8. Ragauskas, A. J.; Beckham, G. T.; Biddy, M. J.; Chandra, R.; Chen, F.; Davis, M. F.; Davison, B. H.; Dixon, R. A.; Gilna, P.; Keller, M.; Langan, P.; Naskar, A. K.; Saddler, J. N.; Tschaplinski, T. J.; Tuskan, G. A.; Wyman, C. E. Science 2014, 344, 12013-12018. $10.1126 /$ science. 1246843

9. Bjørsvik, H. R.; Liguori, L. Org. Process Res. Dev. 2002, 6, 279-290. 10.1021/op010087o

10. Makni, M.; Chtourou, Y.; Fetoui, H.; Garoui, E. M.; Boudawara, T.; Zeghal, N. Eur. J. Pharmacol. 2011, 668, 133-139. https://doi.org/10.1016/i.ejphar.2011.07.001

11. Zhang, S.; Zhang, Y.; Liu, L.; Fang, G. Biores. 2015, 10, 6819-6829. 10.15376/biores.10.4.6819-6829

12. Yoshida, T.; Lu, R.; Han, S.; Hattori, K.; Katsuta, T.; Takeda, K.; Sugimoto, K.; Funaoka, M. J. Polym. Sci. Part A: Polym. Chem. 2009, 47, 824-832.

10.1002/pola.22498

13. Guillon, E.; Merdy, P.; Aplincourt, M.; Dumonceau, J.; Vezin, H. J. Colloid Interface Sci. 2001, 239, 39-48. https://doi.org/10.1006/jcis.2001.7535

14. Qian, Y.; Qiu, X.; Zhu S. Green Chem., 2015, 17, 320-324. 10.1039/C4GC01333F

15. Dong, X.; Dong, M.; Lu, Y.; Turley, A.; Jin, T.; Wu, C. Ind. Crop. Prod. 2011, 34, 1629-1634. https://doi.org/10.1016/i.indcrop.2011.06.002 
16. Sri Ramya, P. V.; Guntuku, L.; Angapelly, S.; Digwal, C. S.; Lakshmi, U. J.; Sigalapalli, D. K.; Babu, B. N.; Naidu, V. G. M.; Kamal, A. Eur. J. Med. Chem. 2018, 143, 216-231.

https://doi.org/10.1016/j.ejmech.2017.11.010

17. Xi, G.; Liu, Z. Tetrahedron 2015, 71, 9602-9610. https://doi.org/10.1016/i.tet.2015.10.080

18. Bagdi, A. K.; Santra, S.; Monir, K.; Hajra, A. Chem. Commun. 2015, 51, 1555-1575. $10.1039 / \mathrm{C} 4 \mathrm{CC} 08495 \mathrm{~K}$

19. Li, L.; Li, Z.; Liu, M.; Shen, W.; Wang, B.; Guo, H.; Lu, Y. Molecules 2016, 21, 49-62. 10.3390/molecules21010049

20. An, W.; Wang, W.; Yu, T.; Zhang, Y.; Miao, Z.; Meng, T.; Shen, J. Eur. J. Med. Chem. 2016, 112, $367-372$. https://doi.org/10.1016/j.ejmech.2016.02.004

21. Hayakawa, M.; Kawaguchi, K.; Kaizawa, H.; Koizumi, T.; Ohishi, T.; Yamano, M.; Okada, M.; Ohta, M.; Tsukamoto, S.; Raynaud, F. I.; Parker, P.; Workman, P.; Waterfield, M. D. Bioorg. Med. Chem. 2007, 15, 5837-5844.

https://doi.org/10.1016/i.bmc.2007.05.070

22. Xi, J. -B.; Fang, Y. -F.; Frett, B.; Zhu, M. -L.; Zhu, T.; Kong, Y. -N.; Guan, F. -J.; Zhao, Y.; Zhang, X. -W.; Li, H. Y.; Ma, M. -L.; Hu, W. Eur. J. Med. Chem. 2017, 126, 1083-1106. https://doi.org/10.1016/i.ejmech.2016.12.026

23. Yang, Y.; Zhang, Y.; Yang, L.; Zhao, L.; Si, L.; Zhang, H.; Liu, Q.; Zhou, J. Bioorg. Chem. 2017, 70, $126-132$. https://doi.org/10.1016/j.bioorg.2016.12.002

24. Jackson, S. P.; Bartek, J. Nature 2009, 461, 1071-1078. $10.1038 /$ nature 08467

25. Lygin, A. V.; Meijere, A. Org. Lett. 2009, 11, 389-392. 10.1021/ol802659m

26. Shinde, A. H.; Srilaxmi, M.; Satpathi, B.; Sharada, D. S. Tetrahedron Lett. 2014, 55, 5915-5920. https://doi.org/10.1016/i.tetlet.2014.08.126

27. Yang, X.; Zhang, P.; Zhou, Y.; Liu, C.; Lin, X.; Cui J. ARKIVOC 2011, 327-337. http://dx.doi.org/10.3998/ark.5550190.0012.a27

28. Yang, X.; Zhang, P.; Hu, L.; Zhang, M.; Liu, C.; Liu, H.; Zhou, Y. Ind. Crop. Prod. 2012, 38, $14-20$. https://doi.org/10.1016/j.indcrop.2011.12.035

29. Yang, X.; Zhang, P.; Zhou, Y.; Wang, J.; Liu, H. Chin. J. Chem. 2012, 30, 670-674. 10.1002/cjoc.201280009 